\title{
A REFERENCE MODEL FOR CONCEPTUALISING THE CONVERGENCE OF TELECOMMUNICATIONS AND DATACOMMUNICATIONS SERVICE PLATFORMS
}

\section{Pasi Kemppainen}

Helsinki University of Technology, Otakaari 1, FIN-02150 Espoo, FINLAND, Tel. +358 9451 2174, Telefax +3589451 5014, E-mail Pasi.Kemppainen@intellitel.com

Key words: Convergence, Network Intelligence, Reference model, Service platforms, IP integration, Telecommunications infrastructure

Abstract: There is no doubt that the telecommunications industry is experiencing a major metamorphosis. Telecommunications, data communications and content from different media and services are converging, resulting in web-based shopping services, mobile interactive banking services and personalised content services, to name a few. This paradigm shift, the convergence, is the driving force of the next generation technologies, services and even businesses. To system vendors and service operators these much-desired new end-user services bring unprecedented business opportunities. However, to bring the best of all these worlds together, there are major technological challenges to meet within the communications network and service infrastructure. And to rationalise and explain the convergence of the existing and forthcoming discrete network infrastructures and services, new conceptualisation approaches are needed. This paper introduces the concept of Telecommunications Network Infrastructure Reference Model (TNI RM) for providing a holistic view for the convergence of telecommunications and datacommunications networks and systems. In addition, the TNI RM presents the importance of content networks in the ongoing convergence.

The original version of this chapter was revised: The copyright line was incorrect. This has been corrected. The Erratum to this chapter is available at DOI: 10.1007/978-0-387-35581-8_35 


\section{INFRASTRUCTURE CONVERGENCE: THE IP INTEGRATION}

\subsection{The Next Generation Telecommunications Infrastructure}

When compared to data communications networks, telecommunications networks are complicated and diversified in nature. Practically, it can be stated that modern data communications networks are relying on IP (Internet Protocol) based technologies. Yet while the underlying infrastructure can be built with IP-independent technologies such as DSL (Digital Subscriber Line) access, SDH (Synchronous Data Hierarchy) ${ }^{8}$ in transport and ATM (Asynchronous Transfer Mode) and Ethernet in switching, IP is the middleware which integrates the whole infrastructure realising the pervasive service network called the Internet.

But telecommunications networks are constructed from a wide variety of protocols and architectures. There are presently no universal middleware protocols to integrate these networks. Telecommunications networks range from WLL (Wireless Local Loop) to cable in access, from IN (Intelligent Networks) to GSM (Global System for Mobile Communications) in valueadded services and from TMN (Telecommunications Management Network) to proprietary systems in management networks. All make use of architectures and signalling of their own. Therefore the telecom technologies are highly segmented, and this is why there still is no single and universal telecommunications service network yet.

According to the Telecommunications Network Infrastructure Reference Model (TNI RM) (Figure 1), telecommunications networks can roughly be divided into seven categories based on their functional and infrastructural characteristics:

- Access networks,

- Transport and bearer networks,

- Switching and signalling networks,

- Service networks,

- Operations and management networks,

- Business networks, and

- Content Networks

\footnotetext{
${ }^{8}$ Also known as SONET (Synchronous Optical Network) in North America.
} 


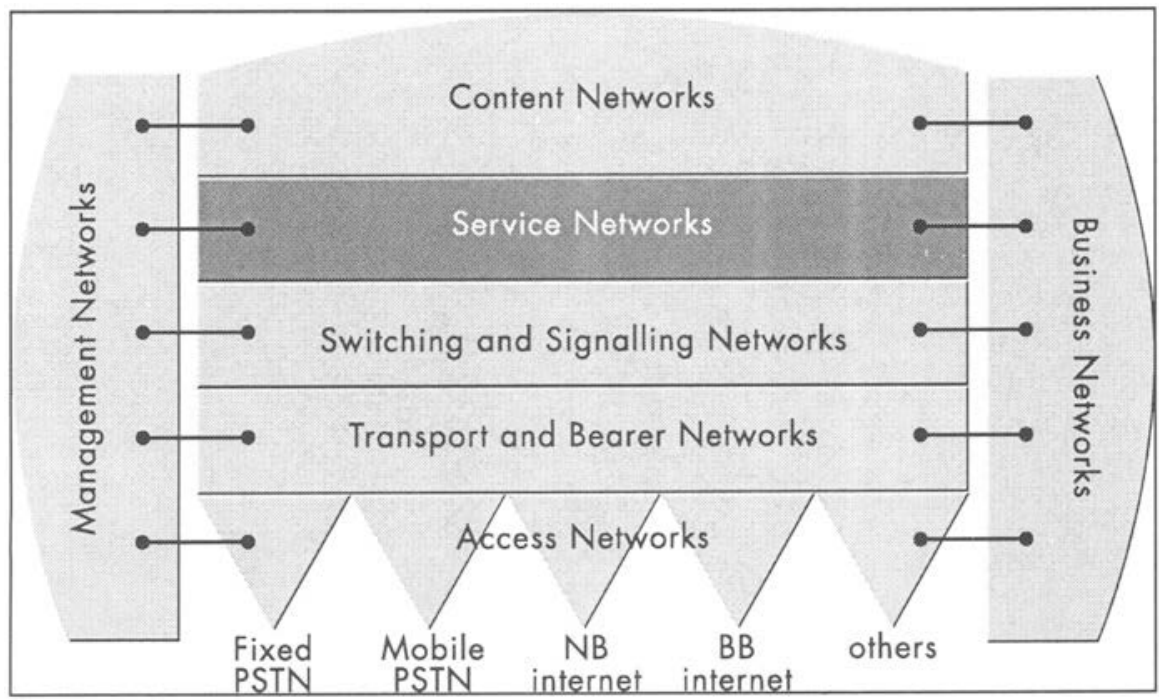

Figure 1. The Telecommunications Networking Infrastructure Reference Model (TNI RM)

\subsection{The Networking Convergence: IP-integration}

Obviously, current telecommunications networks inherently include some or all of aforementioned functionality tightly coupled, as in GSM networks. This is also true in traditional telephone networks (PSTN), where all networking and services (Plain Old Telephony Services, POTS) can be managed by one monolithic, and often proprietary, architecture. However, telecommunications infrastructures and service competition, catalysed by global deregulation and liberalisation, will unavoidably lead to the infrastructure rationalisation, the aforementioned networks will begin to emerge more distinctly.

In fact, to address the infrastructure rationalisation, telecommunications operators are already building their core networks with multiple access technologies ranging from xDSL to cable networks, and implementing mobile network services integrated with fixed network services. Finally different business and management systems are connected to these networks in abundance to realise the necessary business operations (Figure 2). 


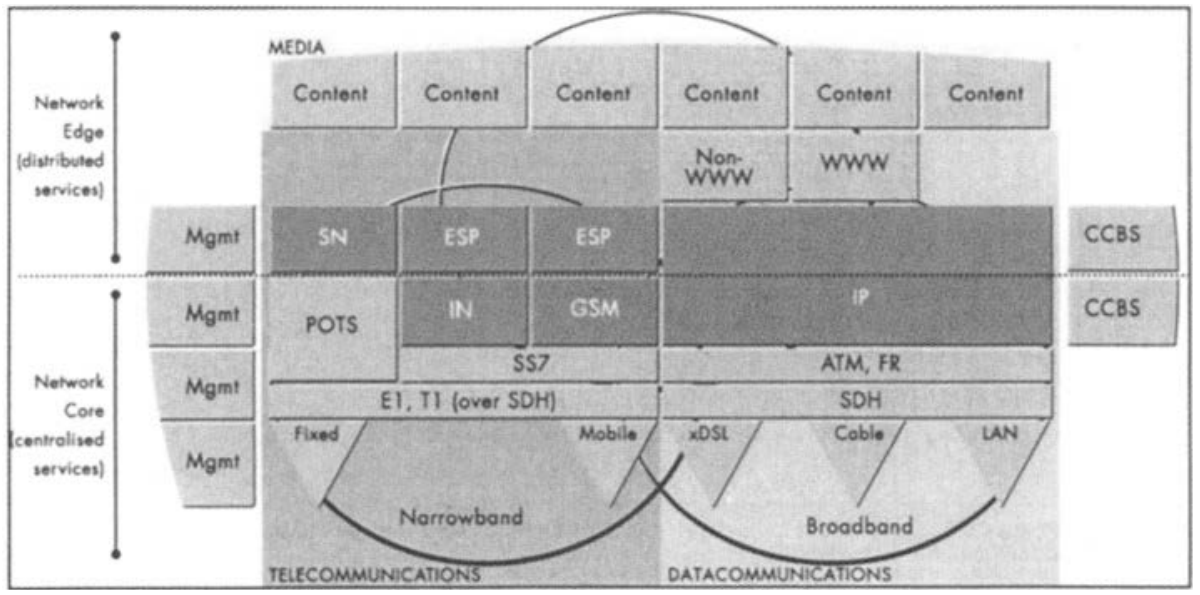

Figure 2. Phase 1 of NI evolution. The networking multiplicity: multiple networks, multiple service platforms, multiple service content formats, multiple business $\left(\mathrm{CCBS}^{9}\right)$ and management systems

It can be claimed that the engine for realising the networking convergence is about making IP technologies the service network middleware for next generation telecommunications infrastructure. However, this is not to say the Internet will replace traditional voice-based telecommunications networks. Instead it will bring new dimensions to communicate ranging from web-based mobile interactive information services based on WAP (Wireless Application Protocol) to multimedia conference services based on the Voice over IP (VoIP) technologies. Therefore, the convergence technologically means the deployment of leading edge Internet technologies and architectures, which can be termed as the IP intelligence; first conjointly with the existing telecommunications architectures (Figure 3), and then in the core in the emerging technologies and architectures (see Section 2).

${ }^{9}$ Customer Care and Billing Services 


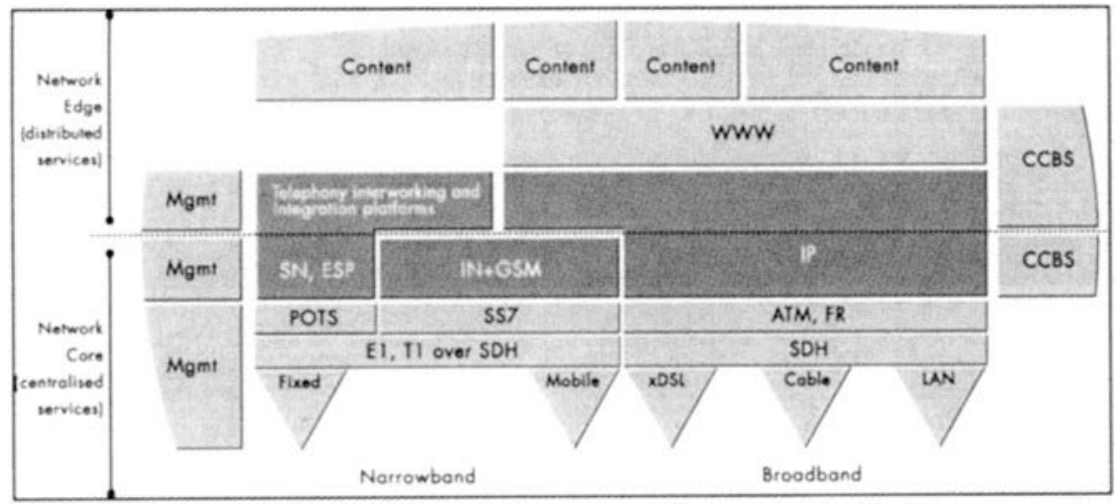

Figure 3. Phase 2. The IP-integration: WAP, VoIP, GSM-IN -integration (CAMEL ${ }^{10}$ ), XML ${ }^{11}$ based content provisioning, content aggregation (portals and hubs)

The outcome of the IP integration will be the emergence of highly sophisticated and ubiquitous services connecting the disparate networks by utilising IP technologies. To further address and describe the consequences and challenges of this IP dominated infrastructure revolution, we will introduce the concept of the Network Intelligence (NI).

\section{SERVICE PLATFORM CONVERGENCE: THE FRAMEWORK OF NETWORK INTELLIGENCE}

\subsection{Network Intelligence: What is it?}

Despite its relatively recent discovery, the framework and paradigm of Network Intelligence (NI) infrastructure has gained different emphases. The common factor in these emphases has been that they concentrate basically on the service platforms. These platforms neither implement transport nor switching architectures, nor do they specify management or business operations networks. In this way, Network Intelligence, as the name implies, is all about making the present and future converged services more intelligent by integrating separate networking paradigms. In addition, the concept of NI explains new complex networking inter-service functionality and interfacing, such as brokering and agent-based systems, resulting in more proactive and autonomous converged networks. Therefore, it can be

\footnotetext{
${ }^{10}$ Customised Applications for Mobile Enhanced Logic
}

${ }^{11}$ eXtensible Markup Language 
said that NI applies a synergestic approach to the convergence: it integrates the networks at the services level, but the outcome is bigger than the sum of its parts.

The objective of the NI integration is based on the fact that as telecommunications and Internet-based services converge to become more access independent, the service platforms must adhere to this trend as well. These NI platforms concurrently support multiple protocols and network interfaces and can simultaneously control multiple converged services (Figure 4). Example of NI integrated service will be GPRS based services, which require the integration of Internet-based switching and GSM based call control and subscriber management.

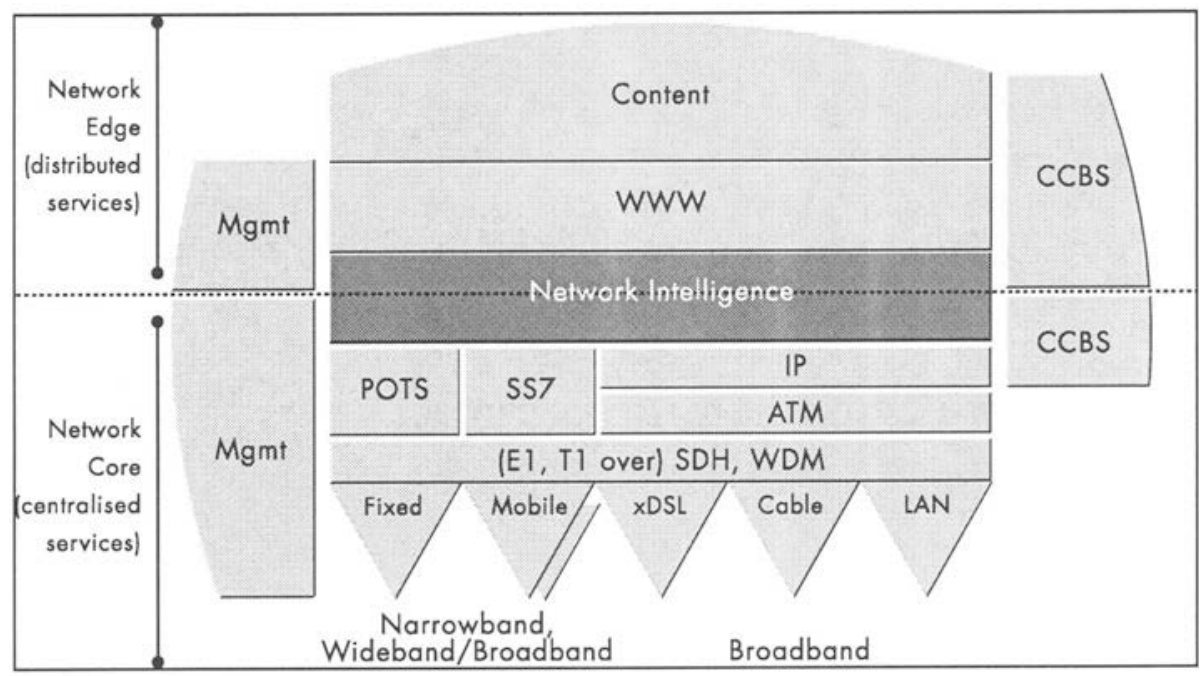

Figure 4. Phase 3. Network Intelligence integration, or service platform consolidation: WWW-based multicontent services with multiple access, converged services billing, GPRS, Network Intelligence Middleware solutions

Although the NI integration will enable seamless service provisioning over multiple access networks, this is not enough. Services still require multiple layers to interface with, such as SS7 and IP based interfaces in signalling and PSTN and ATM based interfaces in switching, and the service platform itself must control the signalling and switching integration. For example, the VoIP gateways integrate the telecommunications and IP networks by low level packet conversions, and adapt the call control and database access across the networks.

The key driver and core component for the inevitable infrastructure consolidation is expectedly the IP (Figure 5). In fact, there are technologies 
available to integrate the PSTN and SS7 networks with IP based services such as the SUN JAIN (Java Advanced Intelligent Network) [1] interface specification for SS7 Java component encapsulation and British Telecom Parlay [2] interfaces for third party IP based PSTN services. Furthermore, in the emerging next generation telecommunications standards, UMTS (Universal Mobile Telecommunications Service) [3] and TINA (Telecommunications Information Networking Architecture) [4] in particular, service platforms are inherently based on IP. So, the ultimate end-result of the service platform consolidation will be a relatively clean convergence of telecommunications and IP technologies and networks bringing about a universal service and content control for multiple access networks, and thus appliances.

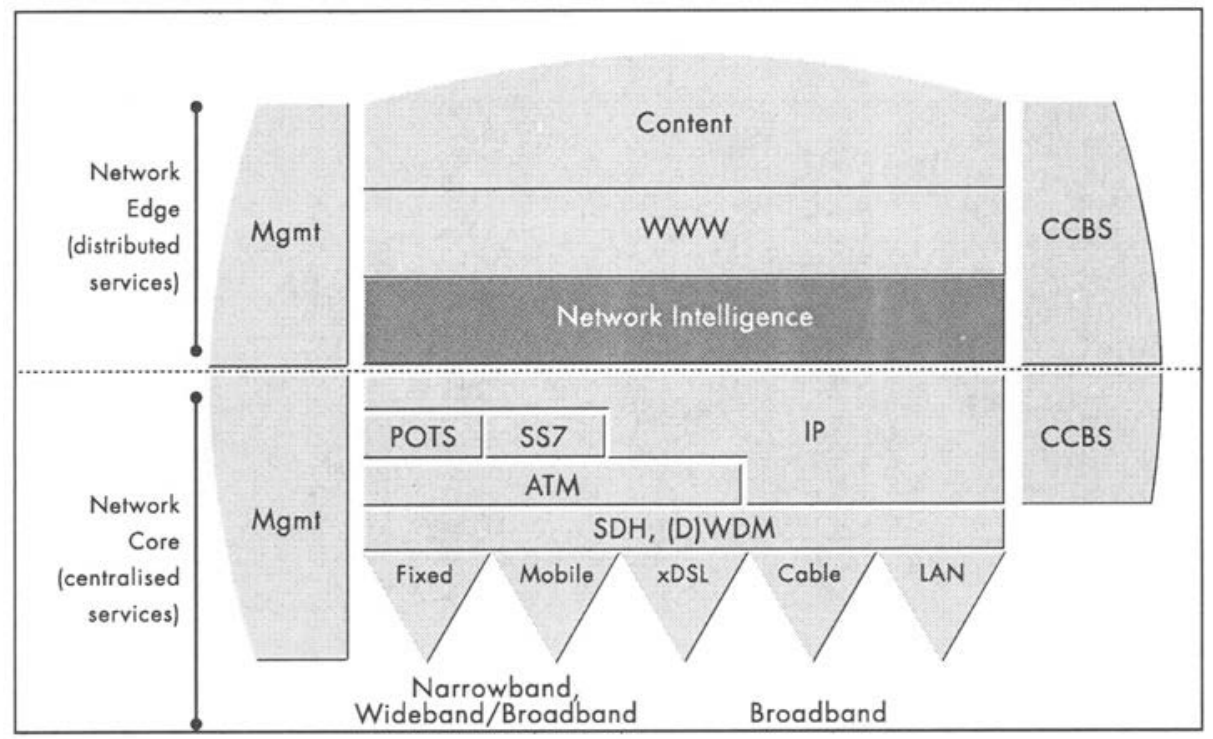

Figure 5. Phase 4. UMTS and TINA: IP-based networking interfaces, Internet-based converged service deployment, spontaneous networking such as IBM T Spaces [5] and SUN Jini [6], agent and brokering technologies

However, though there are service platforms featuring some of the required integration functionality as mentioned, their emphases vary quite widely. The next Section discusses the characteristics and emphases of the present and emerging service platforms catalysing the convergence. 


\subsection{Network Intelligence Platform Characteristics}

For the NI integration, at least three different yet complementing service platform emphases (in addition to the IP based platforms) can be introduced:

- Telecommunications, IN and GSM in particular, enhanced value added services platforms (emphasis on market differentiation) (Evolution Phases 1 and 2)

- Telecommunications and Internet-based telephony interworking and integration platforms (emphasis on unified messaging and overlay switching) (Phases 2 and 3)

- Telecommunications and Internet service infrastructure consolidation and convergence platforms (emphasis on NI integration) (Phases 3 and 4).

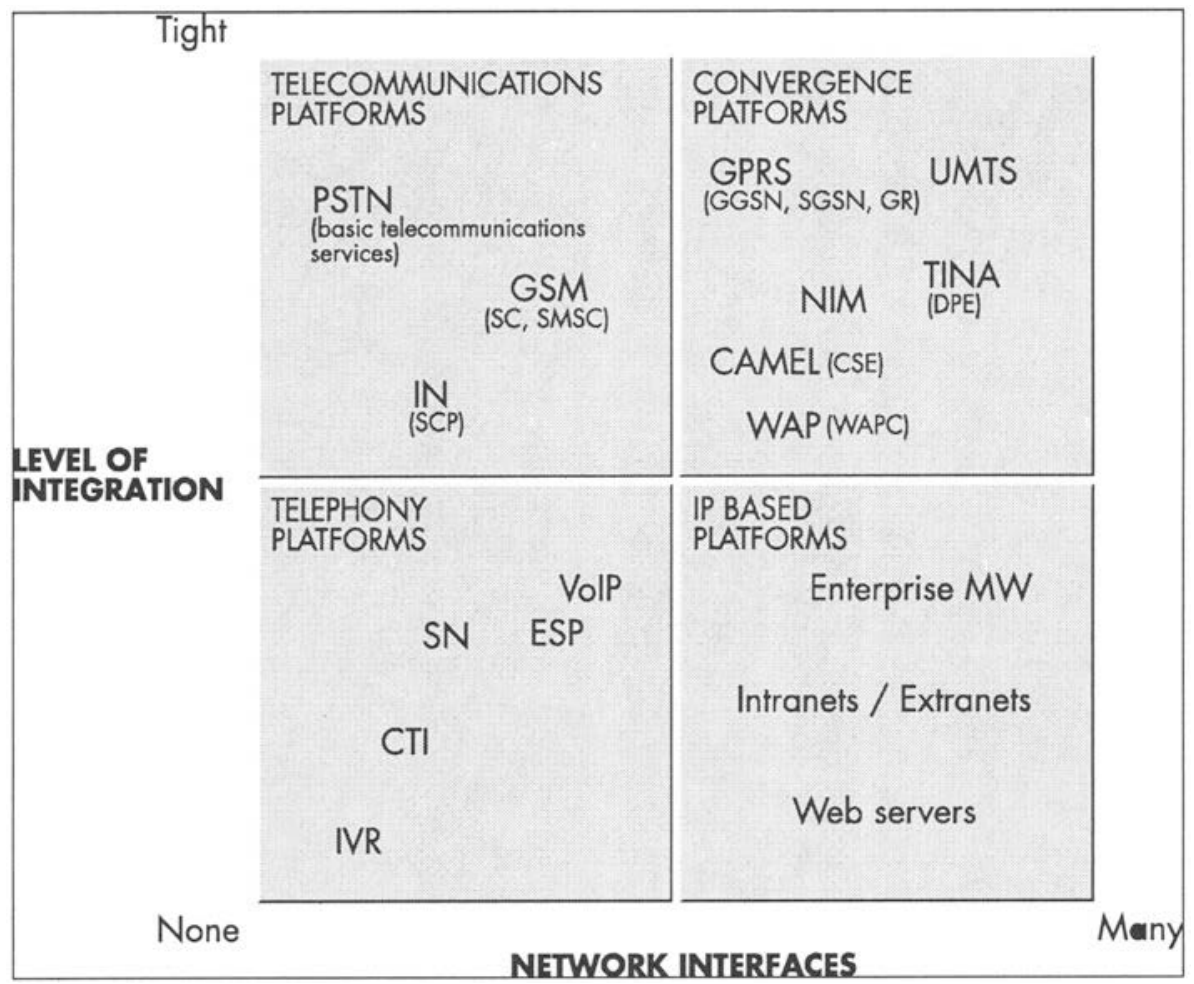

Figure 6. The NI platforms by category.

As presented in Figure 6, the paradigm of NI is not about IN network functionality integrated within GSM systems, nor is it plainly Internet-based 
telephony solutions extended with telecommunications network interfaces. It is an overall framework for provisioning present and next generation telecommunications services seamlessly in fixed (including the Internet) and mobile networks.

The challenge then is not to divide and conquer all of the service networks, but merely having the ability to understand and interface with the present and future telecommunications network systems and services.

\subsubsection{Value Added Services Platforms}

Originally, the concept of Network Intelligence was coined by IN system vendors in the mid-90's, apparently to differentiate from the traditional IN and GSM systems and services. The primary objective has been to introduce value-added services for operators to attract the customers to subscribe to the premium-priced services and to gain customer loyalty. Good examples of new breed value-added services are enhanced corporate VPN services, like mobile centrexes, and premium priced GSM short message-based services, like banking and news services.

As aforementioned earlier, the foundation of the NI concept was based mainly on the existing IN and GSM systems, employing the traditional switches and service control and creation systems. Later on, the concept had been expanded to integrate the Internet services, particularly WWW, with the basic fixed and mobile network services. The objective has primarily been to enable subscribers to parameterise telecommunications services through WWW.

\subsubsection{Telephony Interworking and Integration Platforms}

Another more recent NI platform emphasis had been introduced largely by Computer Telephony Integration (CTI) and Enhanced Service Platform (ESP)-vendors. The CTI-IN platforms take more pragmatic perspective to integrate the IN networks with the Internet-based CTI- and PSTN-based IVR-systems (Interactive Voice Response), such as Service Nodes (SN), to form unified messaging systems. The ESP-IN integration is mainly based on the overlay switching implemented with commercial programmable switches and service nodes. The objective is to offer cost-efficient and rapid time-tomarket enhanced telecommunications services especially for new fixed network service operators (Competitive Local Exchange Carriers, CLECs) and small and medium sized mobile operators.

The essence of the aforementioned platforms is to integrate the telephony call control and service control functionality. The objectives are basically to 
enable subscribers to control the telephony functions such as the voice mail and faxes from their desktops, and to distribute the telephony applications, such as the email-to-speech conversion, over the Internet. Furthermore services, particularly the SNs or ESPs based enhanced services, are implemented largely in fixed networks for various payment and billing services. However, the most rapid growth has been in mobile services, like GSM prepaid services and fraud control management services.

Perhaps the most touted recent telephony interworking based technology has been Voice over IP (VoIP). The objective of VolP technologies is to enable Internet-based telephony services, including IN-type call routing services. The ultimate goal is to integrate the Internet and telecommunications networks to form universal communications network. Unsurprisingly, various vendors and standardisation bodies have defined different VoIP architectures and services.

The IETF has several working groups focusing on the using the Internet to control PSTN services. The Voice over IP, VoIP group [7] concentrates on voice and multimedia transmission, conversion and control aspects between the networks utilising the ITU-T H.323 standards. The PSTN/Internet Interworking (PINT) group's [8] objectives are to define and recommend the usage of the Internet to control PSTN services. The group is focusing on defining PINT-services based on the special client-server architecture and protocols [9].

Another VoIP approach by IETF is being undertaken within the SIP (Session Initiation Protocol) working group [10]. Its objective is to enable the call session management from WWW browsers. The SIP framework simplifies the call control (such as ITU-T Q.931 and H.235 specifications) and session management to become lightweight and WWW compliant. Its protocol is based on the traditional URL-notation (e.g. sip://+999-1234567@net2ph.com) and it interoperates with traditional call control protocols.

To overcome the disparate and proprietary VoIP implementations companies and organisations have already launched projects to harmonise and uniform VoIP architectures and protocols. ETSI has established project TIPHON (Telecommunications Internet Protocol Harmonisation Over Networks) [11] to consolidate the different VoIP emphases. Its objective is to support the market for inter-operator voice communications and related voiceband communications (such as facsimile) between users. Some major vendors have further utilised the TIPHON work to define the OSP (Open Settlement Protocol). The objective of the OSP is to make operator-specific VoIP networks interoperate.

IETF is, on its behalf, currently specifying MGCP (Media Gateway Control Protocol) standard which integrates existing VoIP and media 
switching protocols such as SGCP (Simple Gateway Control Protocol), IPDCP (IP Device Control Protocol) and SIP to form universal framework for interoperable Internet telephony architectures [12].

\subsubsection{Service Infrastructure Consolidation and Convergence Platforms}

The latest and most radical definition for Network Intelligence has arisen from the need to realise multi-networked value-added telecommunications services. The objectives of these platforms are to consolidate the telecommunications and Internet infrastructures and technologies to form an open and universal service network environment, Network Intelligence. At present, this means basically consolidating fixed (IN, PSTN, cable TV), mobile (GSM, PCS [Personal Communications Service]) and Internet (WWW in particular) service environments to form universal and consistent platforms for service design, creation, control and management.

One of the first concepts to propose the aforementioned objectives was TINA (Telecommunications Information Networking Architecture), which literally re-defined the networking architecture and telecommunications service distribution. Although the TINA vision is state-of-the-art, it is utterly complex. Therefore, it is not anticipated that full TINA-based networks will be implemented, but instead, applicable parts of the visions and architectures will be included in next-generation networks. One of the most important TINA architectures is the DPE (Distributed Processing Environment) [13] which defines IP-based distributed service networking environment for next generation converged networks.

Yet a more awaited and important standardisation for the NI integration is UMTS (Universal Mobile Telecommunications Service). The UMTS vision will ideally bring about the integration of wideband and broadband ubiquitous mobile networking with Internet services. UMTS will focus on building on existing and emerging GSM and Internet standards thus being an evolutionary rather than revolutionary standard.

Fortunately, the mobile telecommunications-Internet networking integration is underway with the introduction of the GSM Phase 2+ GPRS architecture. Also, while another GSM Phase 2+ standard called CAMEL (Customised Applications for Mobile Enhanced Logic) [14] already defines the IN-GSM service interoperation (and hopefully leads to a fixed mobile service platform consolidation), GSM truly offers an attractive environment to implement fullblown wideband mobile-based NI functionality in the near future. 
In addition, a generic approach to the networking consolidation is the Network Intelligence Middleware (NIM) [15] based technologies. Their objectives are seamless interoperation of disparate networks and systems, and it combines the NI paradigm with the paradigm of middleware technologies. The NIM technologies will at the same time be flexible towards fast changing business needs (services, charging, customer care), guarantee mission critical integration to production networks and systems, and control the services in a reliable environment.

\section{CONCLUSIONS}

The convergence is undoubtedly revolutionising the communications technologies and business, merging networks of different types and companies of different origins. The future of the telecom business depends on how well old solutions can be combined with new ones to serve new customer needs.

It is obvious that a new breed of technologies will emerge to solve the fundamental differences between telecommunications and Internet technologies, and to integrate the present and future online content services. The characteristics of these technologies are far beyond what has now been already deployed: they will be based on open platforms, they will be multiinterfaced, they will be distributed in nature, they will be componentised yet integrated, they will interoperate autonomously and spontaneously, and finally, they will be reliable and scalable performance-wise.

It is neither easy nor convenient to try to predict what the dominant designs of the converged era will be, but some trends can be observed as described in this paper. Clearly, the most important trend of all is the IP integration and the technologies which have emerged and will be based on the IP foundation such as WWW, Java, XML, WAP, GPRS, NIM and UMTS. This integration takes place mainly in the service and switching layers, and thus accelerates the adoption of leading edge data communications technologies such as gigabit ethernet, WDM, ATM and VoIP. Another trend is the overall integration of the access and transport network interfaces: there will be various different appliances and thus access networks, but they will be tightly integrated to each network operator's terabit core transport network.

At the same time it is not only the networks and services which are converging. Appliances from different vendors are increasingly multifeatured including voice and productivity applications (scheduling, address book, word processing, spreadsheet, WWW browsers, etc), wireless data connectivity and other sophisticated capabilities. It is clear that there 
will be a further consolidation in the technologies in this area. Besides these technologies, there will be the need for new business models and earning logics for the next generation converged businesses. In future, the present customer-supplier relationship and value chains will be totally redefined as has happened in Internet based commerce.

\section{REFERENCES}

[1] Hurley, Hanna, See JAIN Run, Internet Telephony Feb’ 1, 1999. $\mathrm{http}: / / \mathrm{www}$.internettelephony.com/archive/2.1.99/isnews.htm

[2] Parlay Homepage, http://www.parlay.org/default.htm, January 1999

[3] Draft UMTS 23.01 v0.1.0, Universal Mobile Telecommunications System (UMTS); General UMTS Architecture, September 1997.

[4] Eurescom, CORBA as an Enabling Factor for Migration from IN to TINA: A EURESCOM-P508 Perspective. OMG telecom domain taskforce papers. 1998. ftp://ftp.omg.org/pub/docs/telecom/97-01-01.pdf

[5] Wyckoff, McLaughry, Lehman and D. A. Ford, T Spaces, http://www.almaden.ibm.com/journal/sj/373/wyckoff.html, April 1998

[6] Jini Connection Technology Homepage, http://www.sun.com/jini/, May 1999

[7] IETF VoIP Group Homepage, http://www.ietf.org/voip/, August 1998

[8] IETF PINT Working Group Homepage, http://www.bell-labs.com/mailing-lists/pint/, August 1998

[9] Faynberg and Lu, Minutes of the PSTN/Internet Interworking (pint) BOF Meeting, April 1997

[10] Handley, Schulzrinne, Schooler and Rosenberg, Internet Draft SIP: Session Initiation Protocol, http://www.cs.caltech.edu/ schooler/papers/draft-ietf-mmusic-sip-12.txt, January 1999

[11] Tiphon Homepage, http://www.etsi.org/tiphon/, April 1998

[12] Schulzrinne and Rosenberg, The IETF Internet Telephony Architecture and Protocols, IEEE Network May/June 1999

[13] Chapman, Overall Concepts and Principles of TINA, $\mathrm{http} / / / \mathrm{www}$.tinac.com/specifications/documents/overall.pdf, February 1995

[14] DTR/SMG-032320U v0.0.3, Evolution of the GSM platform towards UMTS, September 1997.

[15] Intellitel Communications Ltd, Network Intelligence Middleware White Paper http://www.intellitel.com/whitepaper, April 1999 